\title{
Axiomes de la pédagogie queer
}

Hasheem Hakeem, Université de Calgary

En 2017, alors que j'enseignais dans une école secondaire canadienne, j'ai invité l'écrivain québécois Simon Boulerice pour présenter à mes étudiant.e.s L'enfant mascara (2016), roman qui lie la réalité du meurtre d'une élève transgenre racisée à la réflexion queer sur ce meurtre à travers la fiction. Nous avons étudié ce roman dans le cadre du cours de Français langue immersion $12 .{ }^{1}$ Lors de la conférence, Boulerice a discuté de l'homophobie qu'il a vécue en tant qu'adolescent queer, a expliqué ce qui l'avait motivé à écrire ce roman et a répondu aux questions des élèves. Voulant faire le point sur cette présentation, j'ai rédigé un petit résumé de l'événement pour l'infolettre de l'école, qui est diffusée aux parents d'élèves une fois par mois. J'ai attribué le titre suivant à cette communication : Embracing Sexual Diversity : Montreal author's lived experiences bring novel to life. Avant la publication de l'infolettre, j'ai été convoqué par la direction d'école qui m'a fait part de sa décision de censurer certains éléments de la communication, dont les trois premiers mots du titre appelant à embrasser la diversité sexuelle (voir figure 1).

L'autre passage qui a été biffé faisait référence à l'important rôle que peut jouer le roman de Boulerice dans la prise de conscience de l'homophobie et de la transphobie qui prévalent en milieu scolaire. Selon la direction, les références à l'homophobie, à la transphobie et à la diversité sexuelle faisaient preuve d'un langage « agressif » qui risquait de se heurter à la résistance de « certaines

\section{Embracing Sexual Diversity: Montreal author's lived experiences bring novel to life}

After studying Simon Boulerice's (2016) L'enfant mascara [in English, The mascara child], Grade 12 French Immersion students met face to face with the author via video conference on December $14^{\text {th }}$. L'enfant mascara tells the story of 15-year-old Larry Fobes King, who was murdered by one of his classmates in 2008 at E.0. Green Junior High School in Oxnard, California. The story is based on true events; Larry/Leticia's death is considered one of the most flagrant transphobic crimes in U.S. history. Students had the opportunity to learn more about Boulerice and his inspirations behind writing the novel, as well as his personal struggles growing up gay in Montreal. Boulerice was asked a series of very powerful questions from students, ranging from why Larry/Leticia's story in particular resonated with him, to the importance of writing about sexual diversity and-a range of sexual desires that are socially censured. As an educator who believes social justice to be deeply intertwined with my pedasegic practices, I hope the experience of having both read L'enfant mascara and having dialogued with Boulerice will allow students to become more attuned to the prevalence of homophobia and transphobia in and beyond the classroom, as well as to reflect on the ways-in which they can all become important advocates for LGBTQ rights.

We look forward to hosting Simon Boulerice next semester, but this time for a live book talk in person!

Fig. 1. Infolettre envoyée aux parents, avec les éléments censurés par la direction d'école. 
familles » et de la «population générale » de l'école. La direction a finalement conclu que l'infolettre scolaire ne devrait pas être utilisée à titre de plateforme "émotionnelle» ou « politique » pour militer pour les droits LGBTQ+.

Cette expérience personnelle que j'ai vécue en tant qu'enseignant a déclenché ma réflexion en tant que chercheur universitaire sur les points problématiques de ce type de censure et sur la gestion de la diversité sexuelle et de genre en milieu scolaire. Plus précisément, cette expérience - que je considère comme un exemple révélateur de l'homophobie et de la transphobie institutionnalisées - cristallise pour moi la grande tension entre les lois canadiennes interdisant la discrimination fondée sur l'orientation sexuelle, sur l'identité de genre et sur l'expression de genre, ainsi que les politiques d'inclusion, de diversité et d'équité qu'adoptent et que préconisent inlassablement les écoles, et le fait qu'il semblerait exister dans ces mêmes écoles des mécanismes de censure, d'effacement, de silence et d'invisibilité hétéronormatifs et binaires cisgenres ${ }^{2}$ qui renforcent insidieusement l'homophobie et la transphobie.

Malgré la création de programmes provinciaux voués à contrer précisément cette discrimination, cette tension persiste dans les institutions scolaires de la province. En septembre 2016, le ministère de l'Éducation de la Colombie-Britannique a ordonné aux écoles publiques et indépendantes d'inclure des protections explicites fondées sur la sexualité, l'identité de genre et l'expression de genre dans leurs politiques de lutte contre l'intimidation et dans leurs codes de conduite scolaires. Ces protections sont liées à SOGI 123 (Sexual Orientation and Gender Identity), une série de politiques, de procédures et de ressources pédagogiques élaborées par $A R C$ Foundation, en collaboration avec le ministère de l'Éducation de la Colombie-Britannique et d'autres partenaires, dans le but de promouvoir la valorisation, le respect et l'inclusion de la diversité sexuelle et de genre au sein de toutes les écoles publiques et indépendantes. SOGI constitue un pas dans la bonne direction, certes, mais ce programme, précisons-le, s'inscrit dans une logique de pédagogie inclusive ou de pédagogie de la tolérance. Dans Hétéro, l'école? Plaidoyer pour une éducation antioppressive à la sexualité (2019), Gabrielle Richard souligne les limites d'une telle approche :

Par définition, l'approche par la tolérance pose problème puisqu'elle implique un rapport de pouvoir entre les personnes qui «tolèrent» (et qui sont dès lors détentrices du pouvoir de le faire ou non) et celles qui « sont tolérées » (et dont la légitimité relève du bon vouloir des premières). En aucun cas la pédagogie inclusive ou de la tolérance ne suggère ou ne permet l'abolition de ce rapport de pouvoir qui pose problème en premier lieu. (116) 
D'ailleurs, le programme SOGI s'apparente à une forme de « pinkwashing » dans la mesure où il projette une image d'ouverture et d'inclusion tout en employant un langage très particulier qui considère et ménage les sensibilités d'un lectorat hétéronormé et conservateur (voir Hakeem). Dans cette optique, l'approche SOGI, malgré ses bonnes intentions, finit par maintenir le statu quo hétérocisnormatif, dans la mesure où elle évite de «rendre visibles les dynamiques et les constructions sociales oppressives, de comprendre comment elles en sont venues à exister et à perdurer » (Richard 124). Dans l'anecdote que je viens de raconter, la direction - qui tenait à réitérer son appui envers l'inclusion, la diversité et l'équité - était parfaitement à l'aise avec le message dans la lettre tant et aussi longtemps qu'il appelait de manière générale à la tolérance de la diversité. Cependant, elle avait choisi de censurer toute référence explicite aux enjeux liés à la sexualité et au genre, notamment par rapport à la dénonciation de l'homophobie et de la transphobie en milieu scolaire. Bien que j'aie réussi à résumer l'essentiel de l'événement dans l'infolettre, mon message a dû être hétéronormalisé afin de ne pas perturber le public hétéronormatif à qui s'adressait prétendument la direction. Cette décision montre ainsi les effets pervers de la pédagogie inclusive ou de l'approche par la tolérance, car la défense de la diversité et de l'inclusion ne s'avère que performative, aboutissant au final à la consolidation du statu quo discriminatoire et par conséquent profondément injuste pour d'innombrables élèves, enseignant.e.s et employé.e.s dans les institutions scolaires de la province.

Pour ces raisons, la pédagogie inclusive est inadéquate pour répondre aux besoins des élèves LGBTQ+ dont les réalités demeurent pour la plupart absentes des contenus scolaires (Kjaran ; Rayside ; Richard ; Sadowski). Seule une approche pédagogique queer, qui est une forme de pédagogie critique et antioppressive, réussirait à défier, à bouleverser et surtout à transformer les normes de genre et de sexualité en présence, ainsi qu'à redéfinir la manière dont les élèves appréhendent la diversité sexuelle et de genre. ${ }^{3}$ Afin de conceptualiser cette pédagogie queer, j’ai mené une étude de cas qualitative dans une classe de Français langue immersion du Grand Vancouver pour sonder les perceptions de vingt-quatre élèves de douzième année du secondaire (âgé.e.s de dix-sept à dix-huit ans) au sujet de la diversité sexuelle et de genre, telle que représentée dans une variété d'œuvres queer francophones. Ces perceptions ont été recueillies au moyen d'un questionnaire, d'enregistrements sonores de discussions, de journaux de bord et d'entretiens individuels semi-dirigés. ${ }^{4}$ Cet article a donc deux objectifs : présenter une synthèse des résultats 
de cette recherche et définir une série de huit axiomes de la pédagogie queer. Ces axiomes constituent des principes fondateurs émergeant de l'analyse des données et servant comme guides pour le développement d'une pratique pédagogique queer.

\section{Synthèse des résultats}

Concernant les perceptions générales de la sexualité, les élèves semblent incapables de penser la diversité sexuelle en dehors d'un paradigme identitaire majorité/minorité, c'est-à-dire que l'identité et la sexualité sont liées de manière inséparable et à l'intérieur de ce paradigme, l'hétérosexualité est naturellement majoritaire et normative. Étant donné que ces participant.e.s conçoivent la sexualité sous le prisme de l'identité (nous/eux, normal/pas normal), cette logique catégorielle et hiérarchique les a empêché.e.s de prendre conscience des possibilités ouvertes par la diversité des pratiques sexuelles et des modes de vie d'un individu qui ne sont pas nécessairement rattachés à une identité précise.

En ce qui concerne l'identité et l'expression de genre, une conception binaire, téléologique et biologique est présente chez certain.e.s élèves, et cette conception est parfois utilisée pour délégitimer les réalités trans, non binaires et intersexuelles, ou bien pour qualifier ces dernières de cas extrêmes. Ensuite, il a été particulièrement difficile pour les élèves de penser l'identité de genre séparément de l'expression de genre, ayant souvent tendance à établir un lien logique et naturel entre l'identité de genre d'un individu, et sa manière d'agir (expression de genre) et de vivre (pratiques sexuelles et modes de vie). C'est précisément cette pensée essentialiste qui donne aux élèves l'impression qu'un corps biologique mâle doit correspondre à une identité de genre masculine, doit agir naturellement comme un homme, doit performer une masculinité naturelle et doit afficher publiquement une hétérosexualité incontestable. Cette prétendue cohérence naturelle entre sexualité, identité de genre et expression de genre positionne l'hétérosexualité et le binarisme de genre comme l'unique façon d'agir et d'être au monde et constitue ainsi une extrême réduction du grand éventail de possibilités d'existence queer. D'ailleurs, lorsque la conception essentialiste du genre a été remise en question, cela a provoqué un malaise ou un inconfort chez certain.e.s participant.e.s (notamment chez les garçons), entraînant une attitude défensive que nous pouvons qualifier de « fragilité » (DiAngelo) hétéronormative et binaire cisgenre. ${ }^{5}$ Cependant, c'est grâce à cette fragilité et à la difficulté que les élèves ont eue à saisir la diversité d'expressions de genre, 
notamment incarnée par Ludmilla-Mary (voir figure 2), que j'ai pu constater l'inadéquation du binarisme cisgenre pour rendre compte de la complexité intersectionnelle des êtres humains.

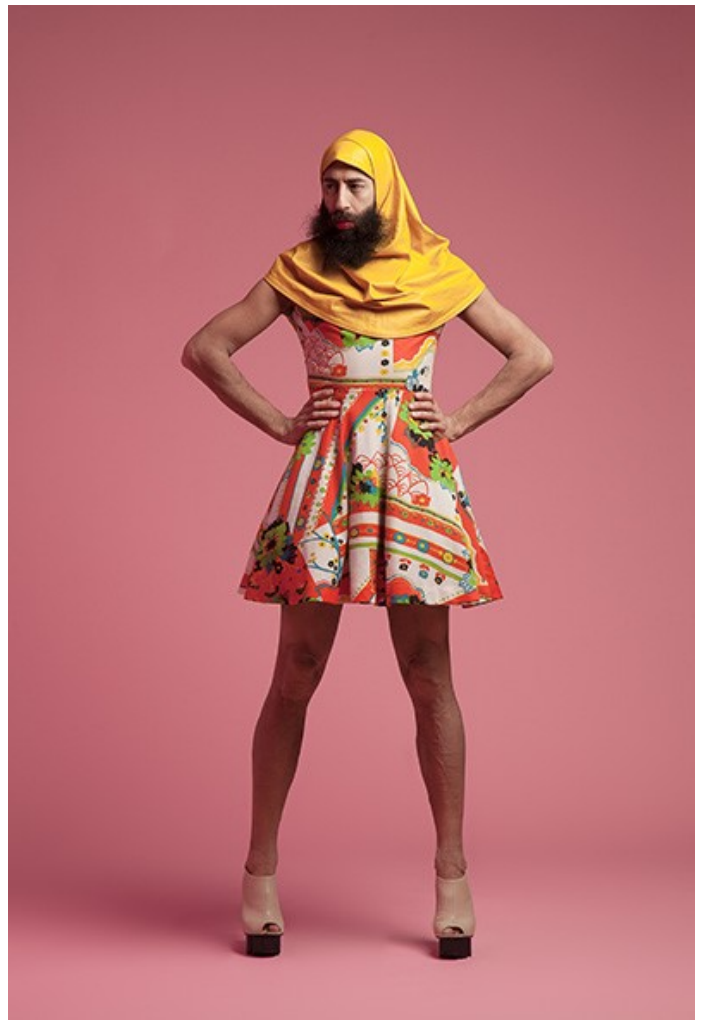

Fig. 2. Ludmilla-Mary, personnage joué par l'artiste-performeur 2Fik. Cette image a été analysée par les participant.e.s.

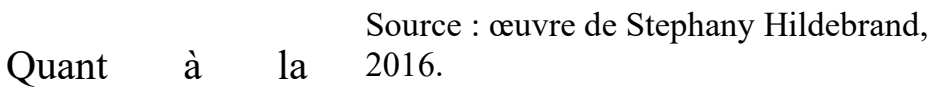

réception

de

l'homophobie et de la transphobie dans les œuvres à l'étude, j'ai été surpris de voir à quel point ces formes de discrimination systémique demeurent fortement incomprises. Pour certain.e.s élèves, l'homophobie et la transphobie relèvent du passé et ne constituent pas un enjeu préoccupant au Canada. Il est pourtant intéressant de constater que la critique de la religion (notamment dans le vidéoclip College Boy ${ }^{6}$ et chez 2Fik) semble avoir suscité plus d'indignation que la violence homophobe et transphobe. Cela pourrait s'expliquer par le fait que dans le discours social, la religion n'est pas considérée comme une position politique parmi d'autres qui devrait être critiquée. Rappelons également que la droite religieuse conservatrice a une forte présence dans l'Ouest canadien. La grande résistance face au programme SOGI en Colombie-Britannique continue à être mobilisée, non seulement par le lobby de certaines associations religieuses, mais aussi par des groupes anti-immigration et suprémacistes blancs (voir MacLeod). 
Un des résultats les plus problématiques de mon étude concerne la performance par certains garçons de la transphobie, voire du mépris, de l'indifférence, de la confusion et de l'ignorance face aux réalités trans. Ces émotions s'inscrivent dans ce que j'appelle une performance sociale de la transphobie, qui, elle, semble être liée à la construction de la masculinité " hégémonique » (Connell) dans l'espace public. ${ }^{7}$ Autrement dit, c'est en performant publiquement ces émotions transphobes - que nous pouvons considérer comme des actes d'expression et d'affirmation publique de formes de rejet des personnes trans dans l'espace social - que certains garçons légitiment leur masculinité hétéronormative et cisgenre pour les autres membres de leur groupe. C'est cette performance de la transphobie qui fonde, dans l'espace public et privé, l'ordre affectif hétéromasculin cisgenre qui est présenté comme étant un «commun » nécessaire et obligatoire. La performance sociale du rejet public de l'identité trans est donc préétablie par cette communauté imaginée hétérocisnormative, pour reprendre l'idée de Benedict Anderson dans Imagined Communities : Reflections on The Origin and Spread of Nationalism (1983). La transphobie semble donc faire partie des règles et des limites qui doivent être intériorisées pour construire l'identité masculine. ${ }^{8}$

Malgré les problèmes rencontrés, toutes les œuvres queer à l'étude ont été particulièrement efficaces pour engendrer chez certain.e.s participant.e.s une prise de conscience de la complicité des institutions sociales dans la violence homophobe, du caractère systémique de la transphobie et d'autres formes d'oppression, de l'importance fondamentale des pronoms pour le bien-être des personnes non binaires, et de la nature mouvante, instable et complexe de l'identité. Il est également important de souligner en dernier lieu le cas particulier d'une participante pour qui la banalisation de l'amour lesbien dans le court métrage J'aime les filles (2016) de Diane Obomsawin l'a profondément touchée en lui faisant prendre conscience de la possibilité d'aimer et de vivre librement sans que ce mode de vie ne soit condamné par la société hétéronormative. L'œuvre est donc porteuse d'une possibilité, car elle anticipe et rend possible un futur que cette participante ne croyait pas possible, comme l'explique d'ailleurs José Esteban Muñoz dans Cruising Utopia : The Then and There of Queer Futurity (2009): «Utopia lets us imagine a space outside of heteronormativity. It permits us to conceptualize new worlds and realities that are not irrevocably constrained by the HIV/AIDS pandemic and institutionalized state homophobia » (35). 


\section{Implications des résultats pour la pratique enseignante : les axiomes de la pédagogie queer}

À la lumière de ces résultats, j'ai développé une série d'axiomes qui pourraient orienter les pratiques enseignantes concernant l'intégration des questions de diversité sexuelle et de genre dans la classe de Français langue immersion 12, ainsi que dans d'autres cours. Dans ce qui suit, un aperçu des axiomes émergeant de l'analyse des données est présenté et discuté.

\section{Axiomes de la pédagogie queer}

1. Il existe une diversité sexuelle, une diversité d'identités de genre et une diversité d'expressions de genre chez les êtres humains, et ces diversités sont intersectionnelles.

2. Il n'existe aucun lien fondamental entre la sexualité d'un individu, son identité de genre et son expression de genre.

3. La diversité sexuelle est normalisée et réduite à l'illusion sociale selon laquelle l'hétérosexualité serait majoritaire.

4. La diversité d'identités de genre est contrôlée à travers un processus de normalisation qui a pour conséquence de la réduire à l'illusion sociale selon laquelle le binarisme de genre serait naturel, et ainsi il représenterait l'unique possibilité de penser le genre.

5. L'homophobie n'est pas identitaire, mais est une forme de discrimination systémique.

6. La construction de la masculinité hétéronormative et cisgenre passe par l'intériorisation et la performance sociale de la transphobie.

7. Quelle que soit leur sexualité, leur identité de genre ou leur expression de genre, certaines personnes, à différents niveaux et à différents degrés, se conformeront à

l'hétéronormativité et au binarisme cisgenre, alors que d'autres s'y opposeront.

8. Le conflit et l'inconfort sont indispensables pour faire de la salle de classe un endroit propice à l'affrontement de nouvelles idées, au développement de la pensée critique et à la transformation des normes dominantes.

D'abord, toute pédagogie queer doit reconnaître l'existence chez tous les êtres humains d'une diversité sexuelle, d'une diversité d'identités de genre et d'une diversité d'expressions de genre intersectionnelles. J'insiste sur l'intersectionnalité de cette diversité, car le but de l'hétéronormativité et du binarisme de genre est précisément de faire croire que les individus ne sont pas intersectionnels, c'est-à-dire qu'ils peuvent être réduits à une seule identité 
unidimensionnelle. ${ }^{9}$ Si tout ce que lisent et visionnent les élèves ne reflète pas la diversité des pratiques sexuelles, des modes de vie et des manières de vivre, d'agir et d'être au monde, ils/elles ne pourront pas prendre conscience du champ des possibilités humaines quant à la sexualité, l'identité de genre et l'expression de genre. Par conséquent, les élèves demeurent pris dans ce que nous pouvons appeler une fatigue hétéronormative, voire dans un état d'épuisement mental et psychologique à force de vouloir correspondre à une identité précise (hétérosexuelle et binaire cisgenre) qui en fin de compte n'est qu'une construction sociale qui sert à les normaliser et à les contrôler. Une pédagogie queer doit ainsi remettre en question la véracité naturelle de toute catégorie identitaire et rappeler qu'il n'existe aucun lien fondamental entre la sexualité d'un individu, son identité de genre et son expression de genre.

Pour combattre la normalisation de la diversité sexuelle et de genre, les enseignant.e.s doivent remettre franchement en question les idées qui renforcent l'homophobie et la transphobie, et qui perpétuent une vision erronée du genre et de la sexualité. Prenons l'exemple des deux préjugés suivants qui sous-tendent les perceptions des élèves: (1) L'hétérosexualité est majoritaire ; (2) Le binarisme de genre est naturel. Si l'on se base sur la réflexion d'Özlem Sensoy et de Robin DiAngelo dans Is Everyone really equal ? (2017), bien que ces idées constituent des opinions, elles ne sont pas du tout fondées sur une connaissance informée sur le genre et sur la sexualité. Si le binarisme de genre est une construction sociale, la notion d'une prétendue majorité hétérosexuelle est, quant à elle, dénuée de toute portée concrète, dans la mesure où les pratiques sexuelles et les modes de vie des individus sont extrêmement variés et ne peuvent donc être englobés par une identité abstraite. L'hétérosexualité en tant que norme constituerait ainsi une invention qui se veut majoritaire dans le but de créer une illusion d'homogénéité au niveau de la sexualité humaine. ${ }^{10}$ Dans une perspective de pédagogie queer, le rôle de l'enseignant.e n'est pas de faire valoir toutes les opinions dans la salle de classe, mais d'amener les élèves à exercer un esprit critique face aux normes, à leur raison d'être, à leur production et à leur reproduction.

C'est notamment ce genre de démarche réflexive qui permettra aux élèves de constater que la catégorie « homophobe » n'est pas identitaire, mais est une forme de discrimination systémique. En d'autres termes, la pédagogie queer ne s'arrête pas simplement à la reconnaissance et à la dénonciation de l'homophobie, mais elle doit travailler pour faire prendre conscience aux élèves du fonctionnement systémique de cette homophobie et de la manière dont les normes en présence contribuent à la renforcer et à la perpétuer. Il est vrai que certain.e.s pourraient dire qu'une telle 
approche est trop politique, certes, mais toute approche pédagogique est politique, alors il est préférable d'être du côté de la réflexion critique au lieu de celui de la répétition aveugle du consensus du moment. Bien que confortable pour ceux et celles qui détiennent le pouvoir et le privilège, ce consensus s'avère extrêmement violent pour les personnes minorisées, car il ne laisse aucune place à la remise en question des idéologies qui sont à la source de leur oppression, de leur exclusion et de leur invisibilité.

Dans le cadre de cette étude, le fait que la construction de la masculinité hétéronormative et cisgenre passe par l'intériorisation et la performance sociale de la transphobie me paraît assez troublant, car cela pourrait suggérer que la masculinité toxique continue à circuler dans certaines écoles et qu'elle est fondée sur la répudiation des personnes trans et non binaires. Expliciter de manière positive dans une pédagogie queer, notamment à travers le choix des œuvres enseignées, que certaines personnes, à différents niveaux et à différents degrés, se conformeront à l'hétéronormativité et au binarisme cisgenre, alors que d'autres s'y opposeront, crée donc un potentiel d'émancipation face à la fatigue hétéronormative de constamment devoir performer un rôle qui va à l'encontre du ressenti sexuel, affectif et psychique des élèves.

Considérant que les perceptions des participant.e.s à notre étude fonctionnent à l'intérieur d'un système discursif dominé par une idéologie hétéronormative et binaire cisgenre, il semble important de réitérer l'un des piliers de la pédagogie queer et critique : c'est-à-dire que le conflit et l'inconfort sont indispensables pour faire de la salle de classe un endroit propice à l'affrontement de nouvelles idées, au développement de la pensée critique et à la transformation des normes dominantes. Ce serait ainsi une grave erreur d'offrir uniquement des cours sur la sexualité où l'on parlerait de diversité sexuelle et de genre dans une perspective de tolérance, car cette approche renforce la vision ghettoïsante des réalités LGBTQ+ et évacue la remise en question de l'hétéronormativité et du binarisme de genre de la discussion. Une pédagogie queer doit plutôt intégrer ces questions de manière transversale afin que cette diversité ne soit pas réduite à une réflexion après-coup, mais qu'elle puisse véritablement déplacer le discours idéologique actuellement dominant sur le genre et sur la sexualité.

Sur un plan plus large, une pédagogie queer serait directement liée à la vision globale des programmes d'études en Colombie-Britannique, notamment en ce qui a trait à la formation de ce que le ministère de l'Éducation appelle le «citoyen instruit». Selon le Ministère, les écoles doivent, entre autres, créer les conditions pour que chaque élève puisse penser de manière critique 
et créatrice, cultiver une bonne image de soi, contribuer pleinement à la société, respecter les autres malgré leurs différences, ainsi qu'être conscient.e des droits de la personne. Autrement dit, pour devenir un.e citoyen.ne instruit.e, il faut que les jeunes soient valorisé.e.s et enrichi.e.s par leur expérience scolaire. Le Ministère explique : «Un système d'éducation de qualité favorise le développement du potentiel humain et améliore le bien-être de chaque membre individuel de la

société britanno-colombienne » (para. 7). Étant donné que la pédagogie queer a pour but de réduire les taux d'abandon, de dépression et de suicide des futures générations de jeunes queer, elle est une condition indispensable à la mise en place d'un système d'éducation de qualité et, donc, à la formation de citoyen.ne.s instruit.e.s, capables d'atteindre leur plein potentiel, de s'épanouir et de contribuer de manière positive à leur communauté, à leur famille et à la société canadienne.

\section{Conclusion}

Le développement d'une pédagogie queer ne peut se réaliser sans la déconstruction de l'hétéronormativité et du binarisme cisgenre qui sont à la source de l'effacement, de l'exclusion et de la censure de la diversité sexuelle et de genre en milieu scolaire. La simple inclusion des représentations de réalités queer dans la salle de classe est déjà un point de départ important, mais ce terme, aujourd'hui galvaudé, perd de son impact dans le milieu scolaire s'il n'est pas au service d'un véritable engagement en faveur de la transformation du statu quo. La pédagogie queer que j'envisage serait profondément ancrée dans ce travail de déconstruction des normes, et non dans une logique de pédagogie inclusive, car si nous suivons la réflexion de Mollie Blackburn dans Interrupting Hate : Homophobia in Schools and What Literacy Can Do About It (2002), les enseignant.e.s doivent non seulement agir en tant qu'allié.e.s des élèves queer, mais en tant que militant.e.s en intégrant de manière critique les réalités LGBTQ+ dans leurs pratiques pédagogiques et en s'élevant contre l'homophobie, la transphobie et toutes formes d'injustices sociales. C'est seulement à partir d'une telle approche critique et antioppressive intégrée de manière transversale dans le curriculum que nous pouvons concevoir, pour reprendre le terme de Gabrielle Richard, une éducation « véritablement démocratique » (12) pour tou.te.s les élèves. La pédagogie queer est donc au service de la défense d'une démocratie pluraliste au sein des institutions scolaires, car cette démocratie pluraliste est l'une des réponses nécessaires aux problèmes culturels, sociaux et politiques auxquels nous faisons face en tant qu'humains aujourd'hui dans un monde de plus en plus globalisé et par conséquent d'une extrême complexité. 


\section{Bibliographie}

Anderson, Benedict. Imagined Communities : Reflections on The Origin and Speed of Nationalism. London : Verso, 1983.

Blackburn, Mollie. Interrupting Hate : Homophobia in Schools and What Literacy Can Do About It. New York : Teachers College P, 2012.

Butler, Judith. Bodies That Matter : On the Discursive Limits of « Sex». New York : Routledge, 1993.

Connell, Raewyn. Masculinities. 2e éd. Berkeley : U of California P, 2005.

DiAngelo, Robin. « White Fragility ». International Journal of Critical Pedagogy 3.3 (2011). 54-70.

Foucault, Michel. Histoire de la sexualité I : La volonté de savoir. Paris : Gallimard, 1976.

Hakeem, Hasheem. « SOGI 123 et son lecteur idéal : une analyse du discours social ». Cahiers franco-canadiens de l'Ouest 32.1 (2020). 61-84.

Kjaran, Jón Ingvar. Constructing Sexualities and Gendered Bodies in School Space : Nordic Insights on Queer and Transgender Students. New York : Palgrave Macmillan, 2017.

MacLeod, Andrew. « BC’s Increasingly Bizarre Anti-SOGI Bandwagon ». The Tyee, 28 mai 2019. https://thetyee.ca/News/2019/05/28/Anti-SOGI-Speaker-Attracts-DubiousCompany/, consulté le 10 février 2021.

Ministère de l'Éducation de la Colombie-Britannique. «Présentation des programmes d'études ». https://curriculum.gov.bc.ca/fr/curriculum/overview, consulté le 10 février 2021.

Muñoz, José Esteban. Cruising Utopia : The Then and There of Queer Futurity. New York : New York UP, 2009.

Pascoe, C.J. Dude You're a Fag : Masculinity and Sexuality in High School. 2e éd., Berkeley : U of California P, 2012.

Rayside, David. « The Inadequate Recognition of Sexual Diversity by Canadian Schools : LGBT Advocacy and Its Impact ». Journal of Canadian Studies 48.1 (2014). 190-277.

Richard, Gabrielle. Hétéro, l'école ? Plaidoyer pour une éducation antioppressive à la sexualité. Montréal : Remue-ménage, 2019.

Sadowski, Michael. Safe is Not Enough : Better Schools for LGBTQ Students. Cambridge : Harvard UP, 2016. 
Sensoy, Özlem et Robin DiAngelo. Is Everyone Really Equal ? 2e éd. New York : Teachers College P, 2017.

Wilchins, Riki Anne. Gender Norms and Intersectionality : Connecting Race, Class and Gender. Lanham : Rowman \& Littlefield International, 2019.

Notes

${ }^{1}$ Ce cours est requis pour l'obtention du diplôme bilingue en Colombie-Britannique.

${ }^{2}$ L'hétéronormativité constitue un système d'oppression qui impose l'hétérosexualité comme étant la seule sexualité ou mode de vie légitime, et qui privilégie les personnes hétérosexuelles en tant que groupe dominant. Quant au binarisme cisgenre, il s'agit d'une idéologie qui catégorise la diversité des identités de genre uniquement en deux catégories (homme/femme) distinctes et immuables. Je qualifie ce binarisme de cisgenre afin d'insister sur le fait que ces deux genres (homme/femme) doivent également correspondre au sexe (mâle/femelle) assigné à la naissance par l'institution médicale.

${ }^{3}$ Pour définir et développer cette pédagogie queer, je m'inspire notamment des travaux de Cris Mayo et de Nelson Rodriguez, de Kevin Kumashiro, de Deborah Britzman, de Gabrielle Richard et de Paulo Freire.

${ }^{4}$ Pour une description détaillée de la méthodologie, voir mon article, intitulé « Vers une pédagogie queer : analyse des perceptions d'élèves de 12e année du secondaire au sujet de la diversité sexuelle », à paraître dans le deuxième numéro du volume 47 de la Revue des sciences de l'éducation.

${ }^{5} \mathrm{Je}$ m'appuie ici sur l'argument de Robin DiAngelo sur la fragilité blanche, qui décrit l'attitude défensive et la défiance des personnes blanches lorsque leurs idées sur les questions de racisme sont remises en cause. DiAngelo développe en plus de détail ce concept dans son livre White Fragility : Why It's So Hard For White People To Talk About Racism (2018).

${ }^{6}$ Réalisé en 2013 par le cinéaste québécois Xavier Dolan, College Boy met en scène, de manière violente et graphique, un jeune garçon queer qui subit l'homophobie dans un internat. La gradation de la violence atteint son paroxysme au moment où le jeune protagoniste est crucifié et abattu par balle par un groupe de garçons au milieu de la cour d'école, alors que ses pair.e.s, les enseignant.e.s, les religieuses et les autorités policières ne font rien pour mettre fin à cette violence. En raison de son contenu violent explicite, le clip a déclenché une grande polémique au moment de sa diffusion, allant jusqu'à être menacé de censure par le Conseil supérieur de l'audiovisuel en France au nom du respect de la dignité humaine et de la protection de l'enfance. La force pédagogique du vidéoclip réside dans sa critique des institutions, notamment scolaires, qui sont présentées comme étant les véritables agents de la violence homophobe.

${ }^{7}$ Si nous suivons la réflexion de Raewyn Connell dans Masculinities (2005), cette performance s'avère presque une obligation, car la masculinité hégémonique " has social authority, and is not easy to challenge openly. One of the effects of hegemony is to shape perceptions of gayness » (156). Ainsi, la masculinité hégémonique dicte aux garçons non seulement les émotions qui doivent être performées dans l'espace public, mais elle façonne également leurs perceptions et leurs manières de penser la diversité sexuelle.

${ }^{8}$ D'ailleurs, dans son étude ethnographique Dude You're a Fag : Masculinity and Sexuality in High School (2012), C.J. Pascoe montre la manière dont l'emploi de l'épithète $f a g$ s'avère être un dispositif disciplinaire afin de contrôler la sexualité et la performance du genre des garçons : «Fag talk and fag imitations serve as a discourse with which boys discipline themselves and each other through joking relationships » (49). Le discours du fag est donc intériorisé et performé par les garçons afin de prouver leur masculinité, et ce, devant le regard masculin hétéronormatif. Par le biais de cette performance sociale de l'homophobie, « boys reminded themselves and each other that at any moment they could become fags if they were not sufficiently masculine » (53). Suivant les conclusions de Pascoe, je peux considérer que, dans le cadre de mon étude, la performance de l'ignorance, de l'indifférence, de la confusion et du mépris face aux expériences trans est ainsi liée à la construction de la masculinité hégémonique. En d'autres termes, la masculinité hégémonique prend forme "through the force of exclusion and abjection, one which produces a constitutive outside to the subject, an abjected outside, which is, after all, "inside" the subject as its own founding repudiation » (Butler 3).

${ }^{9}$ Dans Gender Norms and Intersectionality : Connecting Race, Class and Gender (2019), Riki Anne Wilchins utilise le concept d'intersectionnalité pour souligner la multidimensionnalité et la complexité identitaire des individus : «Intersectionality means that people's bodies and lived experience tend to be more complex [je souligne] than the simple categories or frames we use to understand them » (34). Elle ajoute plus loin : «Intersectionality pushes us to 
widen our frames to see more people, and to also look for more complex identities [je souligne] that fall outside our frame of reference for an issue » (34).

${ }^{10}$ C'est pourquoi dans Histoire de la sexualité I (1976), Michel Foucault s'attaque à la conception d'une identité essentialiste, qu'il considère comme étant au service de la discipline de l'individu et de la régulation de la population (ce qu'il appelle la biopolitique). Pour Foucault, l'identité ne naît pas d'une essence qui la précède, mais émerge des modes de vie et des pratiques de l'individu. Autrement dit, il n'existerait pas naturellement une identité hétérosexuelle, mais plutôt des modes de vie hétéronormatifs qui construisent cette identité. 